\title{
Yihad, islam e inmigración: ¿Choque de civilizaciones o guerra de dioses?
}

\author{
Rafael Arriaga Martínez ${ }^{1}$
}

\author{
Fecha de recepción: 21 de septiembre de 2015 \\ Fecha de aprobación: 11 de enero de 2016
}

\begin{abstract}
Resumen
En este trabajo consideramos la experiencia de los franceses de cultura musulmana a partir de dos visiones teóricas presentes implícitamente en el debate de la inmigración. La primera, asociada estrechamente a la figura de Samuel Huntington, quien postula como irremediable el conflicto entre las culturas y, en consecuencia, la dificultad de integración de los inmigrantes en las sociedades receptoras. La segunda, defendida en el marco del multiculturalismo y otras corrientes de pensamiento, sostiene por el contrario la posibilidad del diálogo, el entendimiento entre los individuos con culturas distintas y la integración de los inmigrantes como un proceso real que avanza al filo de las generaciones. Demostraremos cómo estas dos concepciones de la inmigración se han visto alteradas en su credibilidad con la ola de atentados terroristas sufridos en Francia. En el corto plazo, la credibilidad de la teoría de Huntington ha ganado. Pero, como dice Raymond Boudon, es en los largos plazos que la realidad se pronuncia a favor o en contra de las teorías. Con esta reserva en mente constatamos la existencia en Francia de una percepción cada vez más negativa, tanto de los inmigrantes de cultura musulmana como de las poblaciones con las que se emparentan culturalmente estos inmigrantes.
\end{abstract}

Palabras clave: inmigración, islam, islamismo radical, percepción, guerra de dioses

\section{Jihad, Islam and immigration: A collision of civilizations or a war of gods?}

\begin{abstract}
In this paper, we consider the experience of French people from Muslim culture according to two theoretical views implicitly present in the immigration debate. The first one is closely associated with the figure of Samuel Huntington, who postulated the conflict among cultures as irremediable as well as, consequently, the difficulty of integrating immigrants into the host societies. On the contrary, the second one, defended in the context of
\end{abstract}

Doctor en Ciencias Sociales y Teoría del Conocimiento por la Universidad de Sorbonne-París IV. Profesor investigador en el Instituto de Investigaciones Sociales de la Universidad Autónoma de Baja California (UABC). Contacto: rarriaga@uabc.edu.mx 
multiculturalism and other currents of thought, argues the possibility of dialogue, the understanding among individuals within different cultures and the integration of immigrants as a real process advancing to the edge of generations. We demonstrate how the credibility of these two conceptions of immigration is affected by the wave of terrorist attacks in France. In the short term, the credibility of the theory of Huntington has won. However, as Raymond Boudon says, it is in the long term that reality shows in favor or against theories. With this comment in mind, we find the existence of an increasingly negative perception in France about Muslim immigrants as well as their relatives.

Keywords: immigration, Islam, radical Islam, perception, war of gods.

\title{
Jihad, o islã e a imigração: Choque de civilizações ou guerra dos deuses?
}

\begin{abstract}
Resumo
Neste trabalho foi considerada a experiência dos franceses de cultura muçulmana a partir de dois pontos de vista teóricos presentes implicitamente no debate sobre a imigração. O primeiro, intimamente associada com a figura de Samuel Huntington, que postulou como irremediável o conflito entre as culturas e, consequentemente, a dificuldade de integração dos imigrantes nas sociedades de acolhida. A segunda, defendida no contexto do multiculturalismo e outras correntes de pensamento, argumentam pelo contrário a possibilidade de diálogo, de compreensão entre indivíduos com diferentes culturas e a integração dos imigrantes como um verdadeiro processo avança para a borda das gerações. Demonstramos como essas duas concepções de imigração foram alteradas em sua credibilidade com a onda de ataques terroristas em França. No curto prazo, a credibilidade da teoria de Huntington ganhou. Mas, como diz Raymond Boudon, é nos longos prazos que a realidade se pronuncia a favor ou em contra das teorias. Com esta reserva em mente constatamos a existência em França de uma percepção cada vez mais negativa, seja dos imigrantes de cultura muçulmana como as populações com que estes imigrantes são culturalmente emparentados.
\end{abstract}

Palavras-chave: imigração, Islã, islamismo radical, percepção, guerra de deuses.

\section{Introducción}

Los atentados a las torres gemelas de New York, el 11 de septiembre de 2001, y los ocurridos en París, en enero y noviembre de 2015, en torno a Charlie Hebdo, el Bataclan y otros, son sucesos violentos que favorecen la concepción pesimista de las relaciones entre las culturas que se dan en el marco de las sociedades de inmigración y el mundo. En todo caso, es bajo el signo del conflicto irremediable que Samuel Huntington plantea la relación de 
Occidente con sus minorías. En Estados Unidos son los mexicanos quienes se resisten a asimilar los valores nacionales. En Europa son las poblaciones surgidas de la inmigración proveniente de los países de cultura musulmana. Se trata de un conflicto cada vez más interpretado a la luz de sus obras: El choque de civilizaciones (1998) y El reto hispano (2004).

Es cierto que, en el caso de las ideas de Huntington, casi nadie se refiere a ellas de manera explícita. Quizás porque no es de buen tono apoyarse públicamente en una teoría cruda y contraria a la mirada indulgente con la que, por lo general, se analiza la situación de las minorías en los países de inmigración, una existencia, digámoslo, ya de por sí precaria. No obstante, los conceptos de Huntington flotan en el aire, inspirando a intelectuales, periodistas, políticos y políticas públicas.

Ahora bien, las ideas no son deus ex machina que caen del cielo, tienen un fondo de inspiración epistemológico u hogar que los autores suelen visitar, como cuando un discípulo recurre a su maestro para aclarar una idea, como diría Pierre Bourdieu (1987). Huntington debió haber recurrido a Max Weber, pese a que no lo cita. Apenas si lo menciona, cosa por demás sorprendente por su visión de la cultura y el contacto entre las culturas que se desprende de una cierta lectura de La guerra de los dioses (Weber, 1959), la que pone el acento en la antinomia de valores, en la tensión, el conflicto y la lucha inexpiable. Vamos a ver en qué consiste esa lectura de La guerra de los dioses y cómo se contrapone con la que nos ofrece Raymond Boudon.

Boudon (2012) no niega la dimensión conflictiva de la guerra de los dioses; la admite, pero como una consecuencia lógica de la competencia en la medida que la preferencia por un valor en detrimento de otro afecta intereses y esos intereses, encarnados en individuos, evidentemente, no están exentos de reaccionar incluso de manera violenta. Pero a largo plazo los valores superiores, aquellos que los individuos eligen en el transcurso de un proceso de selección o de racionalización difusa, como diría Weber, ter- 
minan por triunfar. La racionalidad difusa es pues un proceso lento, discernible, pero a largo plazo. La integración de los europeos en Estados Unidos y de los polacos e italianos en Francia ha progresado gracias a este proceso. Y se esperaría lo mismo de los estadounidenses de ascendencia mexicana y franceses de cultura musulmana en sus respectivos países. La lectura que Boudon nos ofrece de la guerra de los dioses no es de lucha a muerte, instintiva; es, por el contrario, racional.

Es con estos dos fondos epistemológicos en mente que vamos a tratar de construir la lógica subyacente al comportamiento de una serie de actores, cuyo papel es clave para comprender la percepción que se tiene en Francia del islam y los problemas con los que comúnmente se le asocia: yihad, inmigración e inseguridad, entre otros. Se trata no tanto de ver cómo la teoría influye consciente o inconscientemente. Se trata más bien de determinar qué actitudes y comportamientos se pueden explicar con una u otra teoría, sin perder de vista aquellos casos en los que es visible la influencia de la teoría en el comportamiento de los individuos. Concebimos la teoría a la manera de un tipo ideal, en el sentido que nos permite determinar y explicar los puntos de convergencia o divergencia entre el pensamiento que encierra la teoría y la acción de los actores. Estos actores, individualizados de manera abstracta, típico-idealizados de manera totalmente esquematizada, son los siguientes: 1) El político que, como parte del gobierno o de la oposición, es llevado a posicionarse en torno a la problemática mencionada. 2) El intelectual que interviene en el medio del debate mediatizado y cuyo auxilio es inestimable para alguna de las partes en conflicto. 3) Lo mismo sucede con los periodistas en el marco de su obligación de informar y opinar; 4) con la opinión pública que se expresa a través de encuestas y sondeos electorales; 5) con el electorado cuya actitud respecto al mismo problema se puede comprender a través del voto.

En cuanto al plano de la realidad en el que actúan todos estos actores, corresponde al de un contexto político e ideológico exacerbado a raíz precisamente de los atentados. De allí el carácter 
acusado del debate en torno a la presencia del islam en Francia y los problemas con los que se le asocia, como es el caso de la inmigración, la seguridad y el orden republicano (las leyes de la república). Hablamos de un debate paroxístico, cuyos efectos deletéreos no merecen ser ignorados.

\section{¿Choque de civilizaciones? ¿Guerra de dioses? ¿Conflicto de valores insoluble?}

Los ataques a las torres gemelas de Nueva York, en 2001, y los sucesos sangrientos iniciados en París con el ataque a las oficinas de redacción de Charlie Hebdo en 2015, nos remiten a una lectura violenta de los contactos entre las culturas. Son hechos suficientemente violentos como para no pensarlos con los conceptos de Huntington, con su famoso choque de civilizaciones (1996). Con Huntington se impone la lectura de una guerra de dioses insoluble y con ello podemos hablar de una teoría exitosa, en todo caso solicitada por los políticos, los periodistas y los analistas ansiosos de surfear sobre una ola portadora, como la que se levanta en Europa y, digámoslo de paso, en Estados Unidos, desestabilizando a amplios segmentos de la población formados de la inmigración. Ese es, evidentemente, el caso de los musulmanes de Europa y, mencionémoslo una vez más, aunque no toquemos el tema, el de los mexicanos o ciudadanos estadounidenses de ascendencia mexicana, cuyas culturas y valores chocan, supuestamente esto, con los de la sociedad receptora. De allí el título por demás sugestivo del choque de civilizaciones y El reto hispano (2004), la otra obra más conocida de Huntington.

Para Raymond Boudon la guerra de dioses no deja de representar un problema, pero no en el sentido que lo plantea Huntington, como algo insoluble, sino como una dificultad llamada a disiparse con el tiempo y en la medida en que los inmigrantes asimilan los valores de la sociedad receptora. Raymond Boudon parte del supuesto de que los individuos disponen de esa facultad extraordinaria que es la de pensar y racionalizar su entor- 
no en función de lo que más les conviene, lo cual presupone a su vez que los valores y lo prescriptivo son materia también de selección, tal como ocurre en el terreno de lo descriptivo. Para Raymond Boudon no puede haber discontinuidad entre el conocimiento ordinario y el conocimiento científico. Existen diferencias, nos dice, pero esas diferencias son de grado y no naturaleza (Boudon, 2003). Y esa es la razón por la que no puede haber lucha impía entre individuos portadores de valores contrarios, porque al final es el razonamiento que invita a los hombres a elegir lo que más les conviene.

De acuerdo con la lógica de la teoría general de la racionalidad, los valores, ideas, creencias o instituciones podrán competir en un momento dado, pero al final unos terminan por ser seleccionados (Boudon, 2003). Es bajo esta perspectiva que habría que comprender la caída del muro de Berlín y la implosión de las democracias populares de Europa del Este y la Unión Soviética: como el resultado de una elección masiva a favor de la democracia liberal. Es cierto que lo que pasa hoy en muchos países de cultura musulmana, exceptuando Túnez, es demasiado confuso como para deslindar el papel y la importancia de los partidarios de la democracia, quienes, como se sabe, han quedado completamente eclipsados por los movimientos surgidos del islam radical. Ahora bien, muchos de estos países son el hogar de las minorías árabemusulmanas que se encuentran diseminadas por toda Europa, y lo que pasa del otro lado del Mediterráneo no deja de tener consecuencias sobre la psique de los que habitan de este lado, como lo puede atestar la existencia de un movimiento yihadista en Europa, constituido por jóvenes europeos que combaten al lado de Daech (acrónimo árabe del Estado islámico) o bajo las órdenes de cualquiera otra organización terrorista activa en Siria e Irak, como es el caso de Djabhat Al-Nosra². Los atentados de París,

\footnotetext{
2 Sin contar los miembros organizados en células djihadistas durmientes basadas en Europa. La Comisión Europea de Justicia estima entre 5.000 y 6.000 los ciudadanos europeos que combaten en Siria en este momento. Se prevé que para fines de 2015
} 
Nice, Copenhague, e Isère, en 2015, y otros sucesos no menos serios, como las agresiones de Colonia en año nuevo, se alinean en el mismo sentido, provocando con ello una gran conmoción y un efecto cognoscitivo permeado con ideas inspiradas en la visión pesimista de Huntington. Si no, ¿de qué otra manera interpretar la multiplicación de los llamados a la cordura por parte de la intelligentsia francesa y alemana, en el sentido de prohibir que se amalgame el islam con el islam radical? La misma interrogante nos tendríamos que formular respecto al desmentís enérgico de un presidente aquí y una canciller allá a todos aquellos que piensan acerca de la incompatibilidad del islam con la democracia y con los valores de la sociedad francesa ${ }^{3}$. Todo parece indicar que el problema ya no es qué tanto les preocupa el islam radical, sino qué tanto se desconfía del islam a secas ${ }^{4}$. Como lo observa Rachida Dati, personalidad de la política nacional de ascendencia argelina, "el islam es un sujeto de angustia" (Le Monde, 7 de julio de 2015), "la angustia de nuestros contemporáneos, angustia al estado puro", diría más amplia y precisamente Michel Houllebecq (Revue de deux mondes, 2015; De Villier, 24 de julio de 2015). Es como si, en el fondo, los franceses cuestionaran el dogma islámico, todo el dogma islámico, no solamente el islam radical.

Se trata de un problema tanto más serio porque el islam en Francia, Inglaterra y muchos países de Europa es percibido como una religión en continuo crecimiento, gracias a la inmigración y la alta tasa de fertilidad de las mujeres musulmanas. De hecho,

la cifra se eleve a 10.000 (Cf. Le Monde, 2015, 13 de abril). En Francia se estima que el número de yihadistas ha venido creciendo de manera literalmente explosiva $(92 \%$ de febrero 2014 a febrero 2015) (Europe 1, 2015).

3 De acuerdo con una encuesta de opinión, realizada por Ipsos/Sopra-Steria para Le Monde y Europe 1, un poco más de la mitad (51\%) de los entrevistados declararon considerar el islam incompatible con la sociedad francesa (Le Monde, 28 de enero).

4 El $74 \%$ de las personas interrogadas en el marco de una encuesta realizada por Ipsos, estima que el "islam es una religión intolerante", incompatible con los valores de la sociedad francesa. El $80 \%$ piensa que la religión musulmana trata de “imponer su modo de funcionamiento a los demás" y más de la mitad piensa que los musulmanes son en su mayoría o en parte "integristas" (Le Bars, 24 de enero de2013). 
en Francia se estima en $8 \%$ la proporción de individuos que profesan la religión musulmana, esto es, 2,1 millones en números absolutos (Simon y Tiberj, 2010) 5 .

Es con este contexto social en mente que el Frente Nacional (FN), tal y como lo expone en su programa político (2015), asocia el problema de la seguridad y la cohesión social a la inmigración. La inmigración es un tema recurrente que se actualiza prácticamente solo con cada atentado o con cada tentativa de atentado abortado por los servicios de inteligencia, como el que acaba de revelar el ministro del Interior, en relación con dos iglesias católicas el 22 de abril de 2015.

Como todos los partidos, el FN busca el poder y lo persigue basándose en estrategias racionalizadas en términos de objetivos y medios, incluyendo las consecuencias que pudiera suscitar el solo hecho de abrir un debate sobre la inmigración, en el medio de un contexto de suma tensión, desconfianza, miedo y enojo frente a la inminencia de un nuevo atentado, y del cual se hablaba desde enero a través de la prensa sin que se supiera en dónde y en qué momento (Le Monde, 2015, 22 de abril). El del Bataclan ocurrió, pese a la movilización febril de los servicios de inteligencia, y se espera otro y, desgraciadamente, otros más. En este contexto de perspectivas sombrías se discute en la Asamblea Nacional la instauración del Estado de Urgencia y la destitución de la nacionalidad a terroristas con doble nacionalidad.

Nada más favorable para que, dentro de este contexto sociocognoscitivo, se dé la transferencia de todas esas emociones inextricables sobre los inmigrantes y sus descendientes, por el solo hecho de compartir con el enemigo que amenaza una misma

\footnotetext{
Se trata de un dato a tomar con mucha reserva. No existen datos fiables en virtud de la ley del 6 de febrero de 1978, llamada "ley de informática y libertades", la cual prohíbe expresamente, en su artículo 8 , la colecta y el procesamiento de datos que revelen "directa o indirectamente los orígenes raciales o étnicos, las opiniones políticas, filosóficas, o religiosas (...) (Legifrance, 2016).
} 
identidad, étnica o religiosa ${ }^{6}$. Pero como las transferencias no se pueden considerar por un solo lado, por su ambivalencia misma, habría que ver entonces quién capitaliza la otra cara, en dónde se encarna la esperanza de un mañana radiante. ¿En el FN?: es lo más probable. Y con tanta más certitud que la transferencia de emociones se ha venido expresando en las urnas con un traspaso efectivo de votos proveniente de los demás partidos: el comunista, el socialista y la Unión por un Movimiento Popular (UMP). ¿Cómo no ver todo esto como el resultado de la desdiabolización, la cirugía a la que ha sido sometido el partido desde que Marine Le Pen tomó sus riendas?

Como se podrá observar, la estrategia del FN funciona y muy bien, como lo demuestran los resultados de las últimas elecciones regionales de diciembre de 2015. Pero si vemos un poco más ampliamente las cosas, nos podremos dar cuenta de que el FN no es el único que sopla sobre las mismas brasas. El Estado islámico y las organizaciones como Al-Qaeda otras organizaciones islámicas hacen lo mismo, pero para que se haga realidad el vaticinio de Eric Zemmour: el caos y la guerra civil. El FN y el Estado islámico comparten una misma visión del individuo y las culturas, y ella corresponde a la que Huntington describe en su teoría. El FN y el Estado Islámico son dos tipos ideales casi puros, dos actores típicos vivos que funcionan con base en la lógica de la lucha inexpiable. A aquellos que piensan la guerra como una posibilidad sin la Unión Europea, Jean Marie Le Pen, fundador del partido, decía, cuando dirigía el partido, que el mejor antídoto para evitarla es el despliegue de la fuerza, que era el mejor medio para

Akhenaton, uno de los raperos más populares de Francia y en particular de los suburbios en donde la representación de los inmigrantes musulmanes es abrumadora, describe en entrevista este proceso de polarización por el que pasa Francia de la manera siguiente: "existe en Francia, una islamofobia que me aterroriza (...) Francia tiene miedo del islam desde septiembre 2001 (...) Es una materia, un tema que no se enseña cuando hay siete millones de nuestros compatriotas que son musulmanes". “QQuiere usted decir -le preguntan- que Francia tiene miedo de su propia población?" "Sí, absolutamente" (Fdesouche, 2015). 
infundir respeto. La actitud de Le Pen, en este punto, aparece teorizada en una recomendación de Huntington: la de no inmiscuirse en los asuntos de los demás. En el fondo, es una ética de convicción la que anima, sobre todo, la línea dura del FN, que no ignora el peligro que significa quebrantar la cohesión social; todo lo contrario, la sopesa, pero como un mal menor, necesario para "salvar a la nación".

La estrategia del FN es duramente denunciada por el primer ministro Manuel Valls como una política orientada a "separar, dividir las poblaciones, los barrios, los territorios. La extrema derecha, los populistas quieren explotar el tema del islam con fines electorales" (Le Figaro, 2015, 28 de junio).

Raymond Boudon observa que las teorías utilitarias se reconocen no tanto porque sean veraces, sino en cuanto puedan ser útiles a los ojos de los individuos o grupos que las promueven. De esta manera, una teoría puede ser útil al Estado-nación, a una clase social o un grupo racial (Boudon, 2012, p. 29). Útil en la medida que, una vez instrumentada a través de una que otra ley o iniciativa, justifica los objetivos que se persiguen, toda vez que se obtienen ventajas de ella. Decimos esto pensando en la teoría de la supremacía de la raza blanca que dio lugar a leyes migratorias de exclusión racial y confesional en los Estados Unidos, entre el fin del siglo XIX y principios del XX. Como no recordar también la intervención en Irak, bajo el mandato de un Bush convencido de encabezar el primer episodio de la guera de las civilizaciones teorizada por Huntington. En Francia, por torpeza o por coqueteo con el electorado de las derechas, Manuel Valls termina por reconocer que "la lucha contra el terrorismo, el yihadismo y el islamismo radical es una guerra de civilizaciones" (Le Figaro, 2015, 28 de junio). ¿Expresión desafortunada, inexacta y excesiva, como lo afirma Julien Dray, secretario nacional del partido socialista? "Que me lo expliquen, declaró en la prensa, ¿cuáles son las civilizaciones que están en guerra? Tengo mis dudas porque yo no creo que la civilización árabe-musulmana sea un riesgo para la civilización cristiana 
(...). Nada más desgraciado que nombrar las cosas con palabras inadecuadas" (Le Figaro, 2015, 28 de junio). Pero, ¿con qué palabras, con qué conceptos describir el reto que enfrenta Francia dentro y fuera de sus fronteras? La dificultad no es menor, como lo demuestran otras tantas declaraciones desafortunadas surgidas al calor de la controversia con el primer ministro, dentro de las propias filas del partido de Valls. Una de ellas es esta: "Valls habla de guerra de civilizaciones: eso es todo lo que esperan yihadistas y Frente Nacional, nos hace perder la guerra cultural". ¿Batalla cultural? ¿Cuál? ¿La que libra Europa dentro de sus fronteras, contra los yihadistas y las mezquitas que, bajo el control de imanes radicalizados, funcionan como verdaderos viveros, incubadoras de yihadistas? ¿O la que libra Occidente en las tierras del islam? Con el enrolamiento del ejército francés en la coalición contra Deach y el apoyo a todos los regímenes árabes que reprimen o controlan los movimientos islámicos, es la que más se confunde con una guerra de civilizaciones, con una guerra contra el islam a secas. Si no, ¿de qué otra manera explicar la actitud conciliadora de Francia con el régimen represivo de Abdelfatah Al-Sisi de Egipto? No toda la relación entre ambos gobiernos gira en torno a los intereses de las grandes empresas que cotizan en el CAC 40 y en particular Dassaut Aviation, que les acaba de vender 24 rafales (Gallois, 2015, 20 de julio). Está en la mesa la cuestión de la cooperación entre ambos países en materia de lucha contra Daech y las actividades terroristas que minan la seguridad de Oriente y Europa. Con un plus que hace soñar a todos los gobiernos con mezquitas en sus suelos: el de revolucionar el islam, separar la fe de la ley, para que pueda cada individuo, con toda libertad, darle a Dios lo que es de Dios y a César lo que es de César. En un discurso pronunciado frente a líderes religiosos reunidos en la universidad Al Azhar de El Cairo, en diciembre de 2014, se explicó que el miedo y el rechazo del islam en el mundo vienen del islam mismo, y que ese temor estaba bien fundamentado (Itele, 2015). De allí el llamado a reformar el islam de manera profunda. 
A los ojos de un público cada vez más amplio, las sutilezas de los políticos para evitar la amalgama entre islam e islam radical no son más que puras peripecias inútiles. La expresión, si confunde, impone la idea de un gobierno inflexible, determinado a deportar imanes radicalizados y cerrar mezquitas, como ya lo ha dicho en la Asamblea Nacional (Le Figaro, 2015, 30 de junio). Valls repite lo que una buena parte del electorado quiere oír.

En los hechos y pese a la presión de las desafecciones partidarias, y el crecimiento del patrón electoral del FN, el gobierno se mantiene fiel a sí mismo. Como lo anota Raymond Boudon (2012, p. 113), la racionalidad instrumental (la que dictan en este caso los intereses ligados a la industria del armamento y la seguridad nacional), puede en algunas situaciones contradecir la racionalidad axiológica (la que dicta en este caso la confianza en el diálogo y entendimiento de las culturas en materia de valores). Ese podrá ser el asunto, tratándose de política exterior pero no de política interior. Porque, como se podrá observar, no es la guerra de los dioses formulada por Huntington -la del conflicto insoluble y de dominación- la que los inspira. Prueba de ello es que en Francia, como en otros países de Europa, se han creado centros de atención especializados para recibir a los jóvenes yihadistas de regreso, después de una estancia de combate en Siria (Le Monde et al. 2015, 29 de abril).Todo lo contrario de lo que espera la dirigencia del Frente Nacional y una buena parte del electorado: la destitución de la nacionalidad y la repatriación. Sí, el presidente Hollande promueve en este momento la destitución de la nacionalidad, pero solo para los binacionales que incurran en actos terroristas.

Tanto en Alemania como en Francia se dice con insistencia que el islam forma parte de la nación, que ha venido para quedarse (Le Point, 2015; Le Figaro, 2010; Le Monde, y AFP, 2015, 15 de junio). Que el islam es compatible con la democracia (Alemagna, 2015, 15 de enero). Que el islam es una cosa y el islam radical es otra, y que si existen vasos comunicativos es porque existen condiciones que los propician, condiciones de ghetización vergonzosas, dignas de 
un apartheid, nos dice Manuel Valls, el primer ministro de Francia (Le Monde, AFP, 2015, 20 de enero). En todo caso, así se expresan los portavoces de los gobiernos, que adhieren a una lectura tolerante de la guerra de los dioses para tratar el yihad promovido por los jóvenes franceses de cultura musulmana.

\section{Islam, yihad e islamofobia: ¿amalgama o polarización?}

Los franceses salen a las calles de París, Lyon y otras ciudades de provincia para expresar masivamente su conmoción y su adhesión a la libertad de expresión ${ }^{7}$. Con todo lo que ello implica, como por ejemplo el derecho de volver a satirizar al profeta $\mathrm{Mahoma}^{8}$, al precio incluso de volver a desatar la cólera de los fieles de Mahoma y en particular la de los yihadistas ${ }^{9}$. De lo cual se puede fácilmente inferir que los franceses no solamente defienden ese valor, sino que además exigen que los demás, los inmigrantes o franceses de origen inmigrante, lo respeten, volens, nolens, guste o no guste ${ }^{10}$.

7 De acuerdo con estimaciones del Ministerio del Interior, las manifestaciones del 11 de enero reunieron en todo Francia a 3,7 millones de personas (2015).

8 Según una encuesta realizada por Ifop para el Journal du Dimanche, el 16 y el 17 de enero del 2015,74\% de franceses con más de dos años de escolaridad después del bachillerato consideraban que, pese al atentado del 7 de enero, se debía continuar con la publicación, "aun cuando algunos musulmanes se puedan sentir heridos o agredidos por la publicación en caricatura del profeta Mahoma". El dato nos parece tanto más significativo porque se refiere a una categoría de la población mayormente representada por franceses de cultura no musulmana. (Otra encuesta, esta vez realizada por Ipsos/Sopra-Steria para Le Monde y Europa 1, encontró que solo 9\% de las personas interrogadas desaprueban abiertamente "la publicación en la prensa de caricaturas satíricas que se burlan de la religión" (Cf. Le Monde, 2015, 28 enero).

9 La revista en cuestión operaba con números rojos la víspera del atentado. Ahora el problema es la administración de una fortuna cuyo monto se estima en más de 10 millones de Euros, véase tanto como dólares (Le Monde, 2015, 19 de febrero). Gracias a la solidaridad de los franceses, la revista vuelve a publicar un nuevo número con el profeta Mahoma en la portada y con un tiraje de siete millones de ejemplares (Jdd, 2015).

10 Se trata de un razonamiento cuya lógica, llevada hasta sus últimas consecuencias, podría encarnar comportamientos como el que anima al diputado holandés Geert Wilders, en su intención de publicar en la televisión caricaturas de Mahoma, bajo el 
Esa es la lectura que se pudo haber hecho desde los suburbios densos y decrépitos en los que vive la inmensa mayoría de los inmigrantes árabes y franceses de cultura musulmana. Y tanto más que eso, de alguna manera, es un eco de declaraciones ya formuladas por el presidente del Partido de los Republicanos, Nicolas Sarkozy ${ }^{11}$. Y es por eso, quizás, que no participaron de manera significativa en las marchas de repudio a los atentados. Más bien brillaron por su ausencia, como dijera el filósofo y ensayista Alain Finkielkraut (2015, 13 de enero).

En efecto, la inmensa mayoría de los manifestantes se vio conformada por "franceses de souche", como osan decir muchos a raíz de la polarización que han provocado los atenta$\operatorname{dos}^{12}$. Muchos otros se quedaron en casa, por temor en algunos casos, y en otros por convicción. Temor a la suspicacia de los demás, a la agresión verbal y hasta física, ¿por qué no? Después de todo, nadie podía garantizar la no presencia en la manifestación de los simpatizantes del Frente Nacional (FN), pese a la consigna de su presidenta de no participar en ella. En lo que respecta a los militantes del partido, los Republicanos (antes UMP) favorables a un acercamiento de su partido con el FN,

pretexto de actuar conforme a derecho, y en particular la prerrogativa de la libertad de expresión. Geert Wilders, como J. Marie Le Pen, se inspira en Oriana Fallaci, nos asegura Lucille Quillet (2015). Fallaci es autora de dos obras muy conocidas La Rage et l'Orgueil (2002) y La Force de la raison (2004)- por la virulencia con la que ataca la presencia del islam en Francia y en Europa. Como Huntington, insiste en el efecto corrosivo, deletéreo para los valores en los que se cimientan las sociedades occidentales.

11 Esa es la lógica de la corriente sarkozista del partido Los Republicanos (antes UMP), cada vez cercana y favorable a un diálogo de entendimiento con el Frente Nacional, partido repudiado por el conjunto de la clase política, incluyendo Nicolás Sarkozy, por sus posiciones extremistas en materia de inmigración e identidad nacional.

12 El mismo presidente François Hollande, para referirse a los franceses de "souche" (de cepa), no supo evitar la denominación, provocando con ello un revuelo en la polémica en torno a la identidad, la temática preferida del Frente Nacional (Boni, 2015), un partido con intenciones de voto a su favor que crece de manera alarmante de elección en elección. Así como François Miterrand le prometió el sótano al partido comunista, Marine Le Pen, la presidenta del FN, se lo promete al partido socialista para las próximas elecciones presidenciales. 
cuya proporción roza el 50\% (Fournier, 2015). Todo ello en un contexto de repetidos actos aislados de repudio a la presencia del islam en Francia. Si no, ¿de qué otra manera interpretar la vandalización de las mezquitas que se registró entre los días del atentado y la manifestación, sin contar que desde entonces se multiplican a lo largo de todo el territorio, como lo atestiguan muchos de los comunicados del Ministerio del Interior? (2015). Por temor pues, pero también, como habíamos dicho, por convicción, como se podrá fácilmente inferir de una reacción sintomática de ello, registrada en las escuelas públicas del país: la de la renuencia de los alumnos de cultura musulmana, en proporción significativa, a participar en el minuto de silencio decretado por el Estado a la memoria de las víctimas de los atentados (Nunès, 2015).

La televisión francesa dio cuenta, muy a su pesar se diría, de esos incidentes, en su noticiero de las 8 de la tarde, la gran misa de los franceses.

Como se podrá ver, no todo fue fraternización durante la manifestación monstruo del 11 de enero. Se respira también el miedo, el desafío ${ }^{13}$ y todo lo que llevan las transferencias negativas en los procesos de polarización ${ }^{14}$, para expresarlo a la manera de Réné Girard (1978). La tensión crece, como si cada quien por su lado rumiara los pronósticos sombríos de un Michel Houellebecq (2015) y Erik Zemmour (2014).

\footnotetext{
13 "Vivimos en un clima de histeria terrorífico, todo el mundo debe calmarse y tener cuidado con los arranques electoralistas", declaró un ex consejero de Sarkozy (Le Monde, 2015,20 de marzo).

14 Emmanuel Todd (2015) se permite incluso dudar de que sea la adhesión y la defensa de la libertad de expresión la que haya inspirado la manifestación del 11 de enero. No es por apego a valores que defienden la libertad de expresión, sino por conveniencia, la conveniencia de que la revista sobreviviera para seguir atacando al islam. Para confortar su tesis, Todd debió haber tomado como datos altamente significativos los siete millones de ejemplares vendidos y los 10 millones de euros colectados, todo ello después de los atentados y las manifestaciones (Jdd, 2015).
} 
Para tener una idea de la magnitud de la sensibilidad de los franceses de hoy respecto de la cuestión de la identidad y la visión dramática de estos dos autores, Houellebecq vendió en tan solo unos meses cerca de 400.000 libros y Zemmour 300.000 solamente en las dos primeras semanas de su aparición en las librerías (RTL, 2014).

Ahora bien, ¿con qué tipo de ideas se confrontan los lectores de estos libros? Zemmour y Houllebecq describen lo que ellos definen como una sumisión por parte del pueblo francés ante el islam. Soumision es el título del libro de Houllebecq, como también es la palabra clave del libro de Zemmour. En Soumision, Houellebecq describe una ficción que se perfila como una realidad muy posible, ya no en el mediano plazo sino en el corto. Los partidos políticos de la derecha y la izquierda se reconocen dentro de un frente común republicano para impedir el triunfo electoral de la extrema derecha. Se trata de una estrategia que ha hecho historia, si recordamos la reelección de Jacques Chirac con una mayoría de sufragios abrumadora en 2002 frente a Jean Marie Le Pen, y se espera que funcione para las próximas elecciones presidenciales de 2017. Los coqueteos en ese sentido se multiplican entre los hombres políticos de ambos partidos después de las duras pruebas de las elecciones regionales de diciembre de 2015, en las que, para vencer al Frente Nacional, el Partido Socialista tuvo que retirar a su candidato y garantizar con ello la victoria de un candidato de derecha, es cierto, pero, al fin, portador de valores republicanos.

Sobre la base de esta actitud, Houellebecq concibe la posibilidad de una alianza de estos partidos con uno nuevo: el de la fraternidad musulmana, todo con el propósito de someter al Frente Nacional en el marco de las elecciones presidenciales de 2022. La existencia de este partido acaba de pasar de la ficción a la realidad, provocando una gran estupefacción entre las filas de los partidos políticos existentes, de izquierda y de derecha, por razones de carácter utilitario -clientela política- pero también de tipo axiológico. 
En el Suicide francés, Éric Zemmour describe al pueblo francés como un pueblo sometido, destruido en su unidad a través de la inmigración. Con un pueblo dentro del pueblo, renuente a vivir "a la francesa", para Zemmour no hay nada más seguro que el caos y la guerra civil (Della Sera, 2014). Zemmour declara esto fuera de Francia y a unos cuantos días de que se produjera la tragedia del 7 y 8 de enero, la manifestación monstruo del 11 de enero y la efervescencia de ideas que desde entonces se publican a través de los grandes diarios nacionales y las editoriales más conocidas. En el contexto del "antes de los atentados", la declaración de Zemmour ultrajaba la moral y la visión oficial de la izquierda y de derecha en materia de inmigración y cohesión social. En "el después" las ideas de Zemmour se banalizan con la multiplicación de sus emuladores, como Thierry Baudet, para quien el "riesgo de guerra civil es real", pero no solo para Francia sino para todos los países de Europa receptores de inmigrantes con cultura musulmana (Baudet, 2015, 24 de abril).

Para Baudet, si no se detiene la inmigración a través del control de las fronteras de Europa, se corre el riesgo de la islamización y la implosión de la unidad nacional, como ocurrió en Irak, Siria y otros países. Cuando una minoría crece es totalmente normal que este crecimiento vaya acompañado de aspiraciones políticas y reivindicaciones de todo tipo.

Una política que no está sostenida por un pueblo dotado de una identidad nacional común, es intrínsecamente inestable. Las diferentes facciones o comunidades perciben desde entonces la política como una competición en la que unos ganan y otros pierden, son ellos o somos nosotros, y se inclinan a tomar el poder cuando el otro es débil. (Baudet, 2015, 24 de abril).

El espectro de la guerra civil se empieza a instalar en la mente de una buena parte de la población como algo enteramente posible, sobre todo en el medio popular, en donde el sentimiento de vivir en estado de guerra conduce a percibir el conflicto en 
términos de ellos y nosotros, de quiénes son ellos y lo que los motiva a declarar la guerra. De acuerdo con estimaciones elaboradas a partir de una encuesta realizada por Ipsos/Sopra-Steria para Le Monde y Europe (2015, 21, 22 de enero) el 53\% de los franceses se siente en guerra después de los atentados del 7 de enero, el $84 \%$ de ellos considera que la guerra es contra el terrorismo yihadista y solo el 16\% piensa que es contra el islam en general. En el medio popular tales distinciones tenderían a desaparecer. Aquí se expresa claramente la idea de que es la motivación de la conquista, la voluntad de imponerse sobre los demás con sus propias leyes (la charia) la que anima al enemigo, un individuo colectivo identificado sin miramiento con el islam a secas (Fourquet y Mergier, 2015, p. 53), puesto que es del islam mismo del que se inspira la charia, la carta magna que rige la moral de los musulmanes. Lo mismo se podría decir de la interpretación belicosa del yihad. El islam es la pandora del yihad. Por más que el presidente y el primer ministro prohíban a los franceses pensar así, Manuel Valls no dice otra cosa cuando exhorta a los representantes del islam de Francia para que cada quien "asuma sus responsabilidades", para que, en otras palabras, denuncien a los "imanes autoproclamados" que apologizan la violencia y el terrorismo (Le Monde, 2015, 15 de junio). Con los atentados del 13 de noviembre de 2015, los parámetros contextuales cambian y con ello la actitud del primer ministro, quien decide en adelante clausurar "las mezquitas y las asociaciones que ataquen los valores republicanos" (RTL, 2015, 16 de noviembre), haciendo valer así las promesas pronunciadas en la asamblea al día siguiente de los atentados del 7 y 8 de enero.

El sentimiento de vivir bajo amenaza, en un ambiente de guerra, decíamos, invita a razonar el conflicto en términos de causa. Y con tanta más razón porque el razonamiento causal no es cosa solo de científicos, también opera en el hombre ordinario. El postulado de la racionalidad nos indica que los actores tienen razones para pensar que son $\mathrm{X}$ e $\mathrm{Y}$ las causas de un estado de cosas, pero esto no implica que esas razones que se forman en torno a 
X e Y sustenten verdades. Esas razones pueden ser frágiles, dudosas o falsas. Ahora bien, hasta donde hemos podido ver, todas las pistas de razonamiento circulan alrededor de la naturaleza de la religión de los otros, del islam, puesto que es en el nombre de Allah que se forman partidos, muyahidines y se cometen atentados sangrientos en países en donde los musulmanes son mayoría o minoría. Son hechos que se propagan a través de los medios de comunicación impresos y cibernéticos. Son noticias de un gran impacto entre el público. Este impacto es emocional, afectivo. En todo caso fue el elemento ansiolítico más importante del año 2014, esto de acuerdo a una encuesta realizada por Ifop para Paris Match el 11 y 12 de diciembre de 2014. La prueba es que el fenómeno mencionado colocó a una buena proporción de las personas encuestadas en un estado de preocupación (86\%) y absoluta preocupación (57\%) (Fourquet y Mergier, 2015). Es cierto que de ningún comportamiento motivado por lo affektuel se podría esperar algo bueno. Digamos esto pensando como muy probable que de esta preocupación se alimente la islamofobia. Pero igualmente podría ser cierto que esta misma preocupación no sea más que síntoma de una actividad racional desarrollada en torno al peligro potencial que significa el islam radical, pensado como una amenaza exógena y endógena. Nosotros nos inclinaríamos a concebir bajo este último ángulo la ideología de la islamización de la que habla Fourquet, independientemente del hecho de que ésta, por otra parte, pueda alimentar a los fóbicos del islam.

La ideología de la islamización invitaría a percibir el islam como una amenaza, como un espectro que surge de cada uno de los grandes temas sobre los que se discute públicamente en los medios de comunicación: inmigración, multiculturalismo, laicismo, inseguridad o seguridad nacional. Es el elemento referencial que permite valorar en un sentido $u$ otro esos mismos problemas. El FN la instrumentaliza a su favor, también la corriente sarkozista del partido Los Republicanos. El Partido Socialista y todos los demás partidos se ven obligados a debatir y tomar posición al respecto. Se podría decir que algo parecido sucede con el indivi- 
duo ordinario, el que forma parte del electorado o de la opinión pública.

Raymond Boudon observa que, para conformar una opinión, los individuos, además de interrogar la realidad, recurren a otras fuentes de información. Una es la que se difunde a través de los medios escritos y visuales, pero, por otra parte, como ya lo hemos dicho, cada quien procesa el problema confrontando la información de la cual dispone con lo que observa directamente, desde una posición -de racionalidad diría Boudon- que no es la misma para todas las categorías sociales. Con esto queremos significar que la complejidad misma de los problemas que plantea la presencia del islam en Francia hace que su comprensión escape al entendimiento de los hombres en proporción variable. Pese a ello, las encuestas de opinión, realizadas a pedido de los medios de comunicación y los partidos políticos, resumen este entendimiento a una opinión expresada con mucha frecuencia a través de un sí o un no, pero de ninguna manera formulada por ellos mismos.

Algo así pasa con los problemas que plantea el FN, los simplifica a la luz de un solo factor: el islam. La brutalidad con la que el FN interroga la realidad se podría resumir en unas cuantas preguntas de tipo:

A. A la vista de las atrocidades que comete Daech y Al-Qaeda en el Medio Oriente y los atentados de enero y noviembre de 2015 en Francia, ¿el islam es o no violento?

B. A la vista de los conflictos en las escuelas ${ }^{15}$, universidades $^{16}$ y empresas ${ }^{17}$, surgidos del desacuerdo de los musulmanes practicantes por razones de inconformidad con condiciones

\footnotetext{
15 Reivindicación del derecho a comida sin carne de puerco en los comedores de las escuelas.

16 Reivindicación del derecho al uso de vestimenta conforme a las exigencias del islam.

17 Creación de salas de oración, adecuación de horarios de trabajo en función de las oraciones, etc.
} 
de vida y trabajo no acordes con la moral y la ética del islam, ¿el islam es o no compatible con el ideal de la laicidad y, de una manera más amplia, con los valores de la república?

C. A la vista de A, B y de los conflictos en los barrios ${ }^{18}$ y en las prisiones sobrepobladas de individuos de cultura musulmana, ¿el islam es factor o no de inseguridad en el país?

D. ¿La inmigración alimenta el crecimiento del islam en Francia, sí o no?

E. ¿Es o no una solución a todos esos problemas el control enérgico de las fronteras y de la inmigración?

No es por puro azar que el FN y el UKPI (United Kingdom Party Independent) del Reino Unido le deban buena parte de su dinámica de crecimiento al tema de la inmigración. En todo caso, esa es la percepción que en buena proporción tienen los franceses (47\%) y los británicos (67\%), según estimaciones determinadas a partir de una encuesta realizada por ICB Research, la agencia Sputnik, entre el 1 y el 4 de mayo de 2015 en Francia, Reino Unido y España (Sputnik, 16 de junio, 2015).

La percepción que se tiene de los acuerdos de Shenguen en Francia y el Reino Unido no es menos entusiasta para estos dos partidos, que no se cansan de exigir su derogación o reafirmar su no adhesión en el caso del UKPI. En Francia son casi 7 personas, y un poco más de 6 de 10 en el Reino Unido, que se pronuncian en favor del restablecimiento del control en las fronteras de los Estados de la Unión Europea y el fin de los acuerdos de Shenguen (Ifop-Le Figaro, 2015a , p.21). Se trata de una apreciación que no solo afecta a las derechas en buena medida. En el Partido Socialista más de la mitad (53\%) expresa, de acuerdo con esta misma encuesta, su adhesión a la supresión del libre tránsito de personas dentro de la Unión Europea. En el Frente Nacional, la

18 Quema masiva de autos en fechas conmemorativas de la identidad nacional, como es el 14 de julio. 
proporción crece a $90 \%$ y a $77 \%$ dentro de las filas del partido de los republicanos (Ifop-Le Figaro, 2015a , p. 23).

La crisis migratoria -la travesía por toda Europa de millones de sirios hacia Alemania y otros países prósperos de Europa del norte- no ha hecho más que degradar el ideal de una Europa sin fronteras. Muchos gobiernos han tomado acta de esta degradación. La prueba es la multiplicación de los controles de sus fronteras, en dondequiera que estas se encuentren: carreteras, campos o ciudades. Pero sin duda alguna lo inédito, lo nunca antes visto desde los tiempos de la guerra fría, son las cercas de malla ciclónica y los rollos de alambre de púas tirados a lo largo de kilómetros de fronteras internas. Pero hay algo más espinoso: el miedo al islam, como podremos verlo enseguida.

En efecto, todo parece indicar que el problema de la inmigración se presenta a los ojos de muchos europeos, y de manera masiva entre el electorado de las derechas, como íntimamente asociado a la inseguridad, entendida esta como una amenaza identificada con el islamismo y el islam. Con el islamismo radical, en la medida que se le percibe como una amenaza a la integridad física de las personas y los intereses de la nación ${ }^{19}$; con el islam, en la medida que, de acuerdo con esta percepción, pone en peligro la transmisión de valores cimentados en el cristianismo. La noción misma de inseguridad cultural parece traducir este estado de cosas. Son muchas las voces que expresan este temor, pero nadie con más crudeza que Nadine Morano, ex colaboradora de Sarkozy y partidaria del diálogo con el Frente Nacional: "La República es laica, pero Francia es un país judeo-cristiano. Tenemos que preservar nuestras raíces, con esto quiero decir que tenemos que controlar la inmigración (...) tenemos que defender nuestra

\footnotetext{
19 Digamos esto pensando solo en las agresiones asociadas a los atentados terroristas, porque el temor en este aspecto tiende a generalizarse. Por lo menos eso es lo que se puede decir después de este incidente lamentable que acaba de ocurrir en el año nuevo de 2016 y que podremos ver más adelante.
} 
cultura (...). La religión musulmana tiene que mantenerse como una religión minoritaria" (Le Figaro, 2015, 11 de julio).

Nadine Morano expresa un miedo que recorre Europa y que solo los partidos de derecha -y de izquierda desvinculados de una base exigua, como es el gobierno de Hollande- tranquilizan con sus políticas de seguridad. El triunfo del PIS (Partido de la Ley y la Justicia) en Polonia, anatemizado por las izquierdas como ultraconservador, nacionalista, católico y xenófobo, no puede ser más ilustrativo (Martínez, 2015). En Alemania, desde Dresde, Pegida no exige otra cosa: que se detenga la islamización de Alemania y se reconozcan las raíces cristianas de Europa. El horror propagado a raíz de las agresiones sexuales colectivas ocurridas en Colonia, en el año nuevo de 2016, termina por doblar la generosa política de asilo de Angela Merkel. Y ello pese al apoyo decidido de una clase empresarial con necesidades de mano de obra, en un contexto de crecimiento económico sin igual en Europa. El hecho es que, contra la racionalidad económica de los empresarios y el pragmatismo de Angela Merkel, basados sin duda, también, en una visión confiada del islam, la canciller cede ante la presión de una opinión pública cada vez más convencida, al calor de los atentados que sacuden a los países vecinos y la llegada de más de un millón de personas de cultura musulmana, del efecto letal del modelo social multicultural para Alemania. Los políticos, al igual que los intelectuales, por pragmatismo o por convicción, siguen ese deslizamiento cognoscitivo del electorado y cuya constatación se expresa a través de lo que intelectuales, politólogos y periodistas califican de derechización de la sociedad (France TV info, 2016, 14 de enero).

En Francia, y particularmente en ciertos medios de comunicación impresos y televisivos, como Le Monde y France 2, se lamentaban del carácter fóbico, irracional, sin fundamento de la asociación en cadena de las agresiones de Colonia con los sirios refugiados y el endurecimiento de la política de asilo de la cancillería alemana. Se trata de un cuestionamiento que, además de poner al desnudo la lógica de los magistrados implicados en el 
proceso, nada ajenos por cierto a las presiones del público alemán, se podría resumir en los términos siguientes: ¿Por qué sancionar la política de asilo a los refugiados sirios, cuando las investigaciones judiciales incriminaban solo a cuatro sirios entre 31 ? ¿Qué importa la nacionalidad, todos eran refugiados y de cultura musulmana!

El proceso es revelador de otra de las razones por las que franceses, alemanes y europeos en general desconfían ya no del islam radical sino del islam a secas, como ya lo habíamos mencionado: la concepción arcaica de la mujer que vehicula y su influencia en la conducta de los musulmanes, con todo lo que ello implica en términos de seguridad para las mujeres. El problema no es menor: se teme que el ataque colectivo a mujeres de Colonia corresponda a una nueva forma de violencia. Con el proceso de Colonia surge un problema que nunca antes país alguno se había planteado, por indulgencia asociada al respeto debido a la dignidad de los inmigrantes o por oportunismo político o por las dos cosas. La racionalidad instrumental y la racionalidad axiológica son tipos puros de conducta, nos dice Weber, en los hechos casi siempre aparecen entrelazados. Pero ahora, con este nuevo contexto cognoscitivo, favorable a las políticas securitarias en detrimento de las libertades individuales, los gobiernos se ven libres para dejar de lado "las pinzas" para tratar a los inmigrantes. Ilustrativo de esto es el programa de educación cívica que el gobierno de Noruega acaba de lanzar para promover entre los inmigrantes el conocimiento y el respeto de las formas liberales de ser de las mujeres danesas. El programa ha encontrado emuladores en Francia y otros países europeos (France2 info, 2016, 18 de enero). Vemos pues como, al final, las agresiones de Colonia favorecen la tendencia a percibir el islam $-\mathrm{y}$ ya no solo el islam radical, como lo habíamos puntualizado antes- en su totalidad como una amenaza a la integridad física de las personas. Se trata de algo que en definitiva mina la confianza y el respeto a las demás culturas, por su capacidad de asimilar los valores republicanos, como dirían los franceses. 
En Francia las diatribas del Frente Nacional en torno al islam indignan al primer ministro Valls porque maltratan un valor en el que muchos creen: el que vehicula la noción de la dignidad humana. Valls declara que hay dos maneras de debatir en torno a la presencia del islam en Francia, "una es con tacto, inteligencia y respeto, lo importante es evitar lo otro: la estigmatización, la fractura, las declaraciones que hieren". Valls aborda el problema con tacto e inteligencia, es cierto, pero no es totalmente por apego al valor, por cuestiones puramente axiológicas. El problema es cómo condenar el fanatismo religioso sin correr el riesgo de ser acusado de islamofóbico, como diría el filósofo estadounidense Michael Walzer (2015).

Se trata de algo que no deja de ser espinoso, aterrador, especialmente cuando se sabe, de acuerdo con una encuesta realizada por ICB Research, que el $27 \%$ de los franceses entre 18 y 24 años admira al Estado Islámico (RT, 2014). Malek Boutih ${ }^{20}$, diputado por el Partido Socialista, lo advierte en su reporte "Génération radicale", presentado recientemente al Eliseo. "La amplitud del fenómeno y su penetración en todos los medios, indican que se podría transformar en un fenómeno de masas" (Malek, 2015). Para un partido cuya sobrevivencia está en juego, el reto es serio. El problema es cómo parar la hemorragia del partido sin herir la susceptibilidad de una comunidad que vota PS de manera masiva. No es pues solo la racionalidad axiológica la que lo inspira en su actitud a seguir respecto de la política migratoria. La racionalidad instrumental, dictada por el interés material (cálculo electoral) juega su parte.

Este no es evidentemente el caso de Marine Le Pen, cuyo comportamiento depende de una estrategia basada en la denuncia de la islamización y la exaltación del sentimiento nacional, sin

20 El reporte de Malek Boutih está basado en una encuesta de campo apoyada en un amplio conocimiento de la vida en los suburbios de las ciudades francesas. Se trata de un conocimiento adquirido en parte por su propia calidad de francés de origen magrebino, y en parte por su posición como presidente de SOS Racisme. 
riesgos para la unidad del partido, dada la casi inexistencia de afiliados y simpatizantes de cultura magrebina.

Le Pen explica los problemas a partir de un esquema de razonamiento de causa única. Los socialistas también, pero para ellos el primum mobile de todos los males es la economía, con el empleo los problemas se absorben. En el poder desde el 2012, las promesas de los socialistas pierden audibilidad. Cerca de la mitad de los franceses (46\%) considera que el FN crece debido a las promesas no cumplidas de los partidos en el poder (Sputnik, 2015, 16 de junio).

Sin experiencia en gobierno y con record virgen en materia de promesas no cumplidas, el FN es el único partido con perspectivas de crecimiento, porque puede hacer creer en lo que promete, como lo hizo el Partido Socialista durante su largo camino al poder. Qué importa que la promesa violente, con el solo hecho de ser creíble, la existencia emocional de millones de franceses de cultura musulmana. Los votantes del FN ya no se avergüenzan de comparecer ante las cámaras de la prensa nacional -como solía ocurrir en las elecciones pasadas precisamente allí en donde el acopio de votos a favor del FN era notable-. Ahora se exhiben y se explican con voz enfadada que votan FN porque "hay demasiados extranjeros e inseguridad". O porque, como decía un elector entrevistado por un diario de izquierda, "ya no estamos en nuestra casa, estamos en la de ellos, ya estoy harto" (Rotman, 2013).

"Hay demasiados extranjeros" (...) "ya no nos sentimos en casa", son expresiones que se han transubjetivizado a lo largo del país. Es una actitud que todos los medios comunican, incluso los más cercanos al partido en el gobierno. En su discurso de clausura del último congreso anual de Frente Nacional, Marine Le Pen promete corregir el problema. Advierte que "estamos en casa". Esto significa que se va a poner en orden la casa, se va a limpiar para que los franceses se sientan en casa. La 
militancia repite en coro "estamos en casa". (Agora Box, 2014, s/p).

Las evidencias de la radicalización de la militancia y la dirigencia del FN no pueden ser más claras. Para nadie es un misterio que los conflictos exacerban la identidad de las partes involucradas en el conflicto, dividen la sociedad en ellos y nosotros. En un contexto surtido de noticias que confirman la montée en puissance del islam, la lógica de la radicalización daría lugar a un razonamiento como este: si ellos se radicalizan todos los días, como lo demuestra la noticia del día ${ }^{21}$, ¿por qué nosotros no podemos adherir a un partido que promete liberarnos de la presencia del islam en Francia, que es una amenaza que pesa sobre nuestras cabezas? Con la noción del yihad y la charia en mente, percibida como la fuente de todas las reivindicaciones pacíficas y violentas, no hay nada que impida la amalgama entre el islam radical, minoritario, y el islam profesado por la mayoría.

Después de todo, se piensa, es el Corán el que ordena la charia y prescribe el yihad. Que existan dos maneras de concebir el yihad, el del alma y el de la espada, no tiene mayor trascendencia. Nadie sabe en dónde, cómo y cuándo alguien abraza el yihad del combate a muerte en lugar de buscar la superación social y espiritual a la que invita el "gran yihad" de la lucha interior.

\section{Conclusiones}

Como dice Raymond Boudon, si X cree en lo que cree, es porque tiene sus razones para creer así. Y si queremos comprender a $X$ entonces tenemos que considerar esas razones para entender por qué creen o piensan así. Tenemos, en otras palabras, que reconstruir la lógica de los actores, considerados estos como actores

21 La del abarrotero que, bajo pretexto de aplicar la charia, atiende a hombres y mujeres por separado (L'Express, 2015, 22 de junio); la del atentado a la fábrica de químicos y la decapitación de un empleador a manos de su empleado, en Isière, el 25 de junio del presente... 
típicos ideales. Para construir un tipo ideal, que no es otra cosa que el proceso mediante el cual se le imputa una racionalidad típica ideal a este actor también típicamente idealizado, debemos, desgraciadamente, caricaturizar los contextos, los actores y las racionalidades. Brutalizamos inevitablemente la realidad para poder capturarla con conceptos. Es solo a ese precio que logramos trazar o poner en relieve el conflicto de valores que polariza la sociedad francesa en este momento, colocando por un lado a los inmigrantes o franceses originarios de países de cultura musulmana y, por el otro, a los franceses que se reclaman de souche, es decir de origen o de cepa. Vemos pues cómo la lectura blanda de la guerra de los dioses abandona a los ocupantes del palacio, frente a una plaza pública cada vez más seducida con la retórica del Frente Nacional en torno a la inmigración y la identidad nacional. En efecto, todo parece indicar que el 11 de septiembre en Nueva York y los demás atentados que han sacudido a Europa mueven las voluntades en la dirección de la visión pesimista y fatalista de Huntington, porque, como se sabe, para Huntington no puede haber diálogo y entendimiento entre las culturas como lo concibe Raymond Boudon, sino choque y confrontación.

La óptica de Huntington no solo hace escuela entre los hombres de ciencia, también se transubjetiviza entre políticos, periodistas y una buena parte del electorado, si tomamos en cuenta la adhesión cada vez más importante de los franceses a las tesis del Frente Nacional, el partido que siempre pierde las elecciones, aun cuando se pueda jactar de ser el primer partido de Francia por el número de votos.

Es cierto que el 11 de septiembre en Nueva York y los demás atentados que han sacudido a Europa mueven las voluntades en la dirección opuesta a lo que se pudiera pronosticar a partir de la teoría general de la racionalidad. Pero no es porque los individuos tengan sus propias razones para creer en la incompatibilidad del islam con los valores de Occidente que tengan razón. Porque esas razones pueden ser aceptables, falsas, frágiles o dudosas. La realidad con su cortejo de hechos se encarga de poner 
en su lugar a las teorías; funciona como un veredicto que cae, pero en el largo plazo. Por el momento y en virtud de lo que nos dice la teoría general de la racionalidad, las razones que asisten a los periodistas, los políticos y los intelectuales que difunden la idea de la incompatibilidad de los musulmanes de Europa con el ideal de la democracia son más que dudosas.

\section{Referencias}

Agora Box (1 de diciembre 2014). Discours de Marine Le Pen - La foule scande "On est chez nous". Agora box. Recuperado de http://www.agoravox.tv/actualites/politique/article/ discours-de-marine-le-pen-la-foule-47837

Alemagna, L. (15 de enero de 2015). Hollande: L'islam est compatible avec la démocratie. Libération. Recuperado de http:/ / www. liberation.fr/politiques/2015/01/15/hollande-l-islam-estcompatible-avec-la-democratie_1181152

Baudet, T. (24 de abril de 2015). Le risque de guerre civile paneuropéenne est réel. Le Figaro. Recuperado de http://www. lefigaro.fr/vox/monde/2015/04/24/31002-20150424ARTFIG00374-thierry-baudet-le-risque-de-guerre-civile-paneuropeenne-est-reel.php

Boni, M. (2015). Hollande reprend l'expression "francais de souche" chère à l'extrême droite. Le Figaro. Recuperado de http://www.lefigaro.fr/politique/le-scan/ citations / 2015/02/24/25002-20150224ARTFIG00108-hollande-reprend-1-expression-francais-de-souche-chere-a-1extreme-droite.php

Boudon, R. (2003). Raison, Bonnes raisons. Paris: Presses Universitaires de France.

Boudon, R. (2012). Croire et savoir. Paris: Presses Universitaires de France.

Bourdieu, P. (1987). Choses dites. Paris: Editions de Minuit.

De Boni, M. (28 de junio de 2015). Guerre de civilisation: Julien Dray (PS) fustige "l'amalgame idéologique" de Manuel Valls. Le Figaro. Recuperado dehttp://www.lefigaro.fr/politique/le-scan/couacs/2015/06/28/25005-20150628ARTFIG00154-guerre-de-civilisation-julien-dray-fustige-1amalgame-ideologique-de-manuel-valls.php 
DellaSera, C. (30 de octubre de 2014). Ilsuccesso di Zemmour,1?arrabbiato anti-élite La Francia si è suicidata. Recuperadodehttp:// archiviostorico.corriere.it/2014/ottobre/30/successo_ Zemmour_arrabbiato_anti_elite_co_0_20141030_57c58d326000-11e4-8dd4-2fb2f9df4f43.shtml?refresh_ce-cp

De Villiers, T. (24 de julio de 2015). Baudelaire, Bernanos, Houellebecq: les écrivains sont-ils des prophètes? Le Figaro. Recuperado de http://www.lefigaro.fr/vox/histoire/2015 /07 / 24/31005-20150724ARTFIG00200-baudelaire-bernanos-houellebecq-les-ecrivains-sont-ils-des-prophetes.php

Europe 1 (2015). La France et ses djihadistes: le phénomène en chiffres.

Recuperado de http://www.europe1.fr/faits-divers/lafrance-et-le-djihad-le-phenomene-en-chiffres-2379613

Fallaci O. (2002). La rage et l'orgeuil. Paris: Editions Plon.

Fallaci O. (2004). La Force de la raison. Paris: Editions du Rocher.

Finkielkraut, A. (13 de enero de 2015). Une bataille s'est engagée entre le parti du sursaut et le parti de l'Autre. Le Figaro. Recuperado de http://www.lefigaro.fr/vox/societe/2015/01 /13/31003-20150113ARTFIG00319-finkielkraut-qu-est-ceque-le-politiquement-correct-un-antiracisme-qui-a-perdula-tete.php?redirect_premium

Fournier, A. (3 de marzo de 2015). Le FN, acteur incontournable des départementales. Le Monde. Recuperado de http://www. lemonde.fr/politique/article/2015/03/03/le-fn-acteur-incontournable-des-departementales_4585762_823448.html

Fourquet, J. y Mergier, A. (2015). Le catalyseur. Paris: Fondation Jean Jaurès.

Fdesouche (14 de marzo de 2015). Akhenaton: Si Marine Le Pen prend le pouvoir, je quitte la France. Fdesouche. Recuperado de http:/ / www.fdesouche.com/575225-islam-akhenaton-lafrance-peur-de-sa-propre-population-video

France TV (14 de enero de 2016). Entrevista con Jean D'ormesson. Recuperado de

http:/ / www.francetvinfo.fr/replay-jt/france-2/20-heures/jt-de-20hdu-mercredi-13-janvier-2016_1255943.html

France 2 info (18 de enero de 2016). Norvège : des cours de respect pour les réfugiés. Recuperado de http://www.francetvinfo.fr/ monde/europe/migrants/norvege-des-cours-de-respectpour-les-refugies_1275305.html 
Gallois, D. (20 de julio de 2015). Livraison des trois premiers Rafale à l'Egypt. Le Monde. Recuperado de http://www.lemonde. $\mathrm{fr} /$ economie/article/2015/07/20/livraison-des-trois-premiers-rafale-a-l-egypte_4690865_3234.html

Girard, R. (1978). Les choses cachées depuis la fondation du monde. Recherches avec Jean-Michel Oughourlian y Guy Lefort. Paris: Editions Bernard Grasset.

Houellebecq, M. (2015). Soumission. Paris: Editions Flammarion.

Huntington, S. (1996). The clash of civilizations and the remaking of the world order. Nueva York: Simon and Shuster.

Huntington, S. (2004). ¿Quiénes somos? Los desafíos de la identidad nacional estadounidense. Barcelona: Paidós.

Ipsos/Sopra-Steria (21 y 22 de enero de 2015). Comment les Français perçoivent l'islam et les musulmans. Recuperado de http:/ / www.francetvinfo.fr/monde/proche-orient/offensive-jihadiste-en-irak/video-comment-les-francais-percoivent-1-islam-et-les-musulmans_809779.html

Ifop-Le Figaro (2015). Les Européens et la question des flux migratoire. Jérôme Fourquet et Alexendre Bourgine, Département Opinion et Stratégie d'entreprises. Ifop-Le Figaro. Recuperado dehttp://www.ifop.com/media/poll/3087-1-study_file. pdf

Itele (12 de enero de 2015). Egypte: le président Al Sissi appelle à "révolutionner" 1'islam. Itele. Recuperado de http:/ / www.itele. $\mathrm{fr} /$ monde/video/egypte-le-president-al-sissi-appelle-arevolutionner-lislam-107359

Jdd (23 de enero de 2015). Charlie Hebdo dépassera finalement les 7 millions d'exempaires. DJJ. Recuperado de http://www. lejdd.fr/Medias / Presse-ecrite/Charlie-Hebdo-depasserafinalement-les-7-millions-d-exemplaires-714256

Le Bars, S. (24 de enero de 2013). La religion musulmane fait l'objet d'un profond rejet de la part des Français. Le monde. Recuperado de http://www.lemonde.fr/societe/article/2013/01/24/ la-religion-musulmane-fait-l-objet-d-un-profond-rejet-dela-part-des-francais_1821698_3224.html

L'Express (22 de junio de 2015). Jours réservés aux hommes et aux femmes: une épicerie musulmane crée la polémique. $L^{\prime}$ Express. Recuperado de http://www.lexpress.fr/ actualite/societe/religion/jours-reserves-aux-hommes- 
et-aux-femmes-une-epicerie-musulmane-cree-la-polemique_1692265.html

Le Figaro (10 de octubre de 2010). Angela Merkel s'adresse aux musulmans. Le Figaro. Recuperado dehttp://www.lefigaro.fr/ flash-actu/2010/10/06/97001-20101006FILWWW00687angela-merkel-s-adresse-aux-musulmans.php

Le Figaro (28 de junio de 2015). Manuel Valls: “Nous ne pouvons pas perdre la guerre de civilisation contre le terrorisme". Le Figaro. Recuperado de http://www.lefigaro.fr/politique/ lescan/citations/2015/06/28/2500220150628ARTFIG000 1 manuel-valls-nous-ne-pouvons-pas-perdre-la-guerre-decivilisation-contre-le-terrorisme.php

Le Figaro (30 de junio de 2015). Le gouvernement prêt à fermer des mosquées liées à l'islamisme radical. Le Figaro. Recuperado de http://www.lefigaro.fr/actualitefrance / 2015/06/30/01016-20150630ARTFIG00320-legouvernement-pret-a-fermer-des-mosquees-liees-a-l-islamisme-radical.php

Le Figaro (11 de julio de 2015). Pétition sur les églises. Guaino et NKM se désolidarsent de Sarkozy. Le Figaro. Recuperado de http://www.lefigaro.fr/politique/le-scan/2015 /07/10/25001-20150710ARTFIG00155-petition-sur-leseglises-guaino-et-nkm-se-desolidarisent-de-sarkozy.php

Legifrance (2016). Le service public de la difusion du droit. Recuperado de https:/ / www.legifrance.gouv.fr/affichTexte.do?cidText e=JORFTEXT000000886460

Le Monde (20 de enero de 2015). Manuel Valls évoque «un apartheid territorial, social, ethnique» en France. Le Monde. Recuperado de http: / / www.lemonde.fr/politique/article/2015/01/20/ pour-manuel-valls-il-existe-un-apartheid-territorial-socialethnique-en-france_4559714_823448.html

Le Monde (28 de enero de 2015). Sécurité, politique, islam: comme réagissent les Français après les attentants? Le Monde. Recuperado de http://www.lemonde.fr/societe/article/2015/01/28/securite-politique-islam-comment-reagissent-les-francais-apres-les-attentats_4564681_3224.html

Le Monde (19 de febrero 2015). "Charlie Hebdo": le casse-tête de la reconstruction. Le Monde. Recuperado de http://www. lemonde.fr/m-actu/article/2015/02/19/charlie-hebdo-lecasse-tete-de-la-reconstruction_4578595_4497186.html 
Le Monde (20 de marzo de 2015). Inquiet de la montée du FN, Nicolas Sarkosy dénonce le système d'intégration francais. Le Monde. Recuperado de http:/ / www.lemonde.fr/electionsdepartementales-2015/article/2015/03/20/inquiet-de-lamontee-du-fn-nicolas-sarkozy-denonce-le-systeme-d-integration-francais_4598315_4572524.html

Le Monde (13 de abril de 2015). Manuel Valls dévoile le nombre de français kamikazes en Syrie et en Irak. Le Monde. Recuperado de http://www.lefigaro.fr/actualitefrance / 2015/04/13/01016-20150413ARTFIG00374-manuel-valls-devoile-le-nombre-de-francais-kamikazes-ensyrie-et-en-irak.php

Le Monde (22 de abril de 2015). Valls: la France face à une "menace terroriste sans équivalent dans le passé". Le Monde. Recuperado de:http: / www.lemonde.fr/societe/article/2015/04/22/ valls-la-france-face-a-une-menace-terroriste-sans-equivalent-dans-le-passe_4620681_3224.html

Le Monde (29 de abril de 2015). La France veut encadrer les "jeunes de retour" du djihad. Le Monde. Recuperado de http:/ / www. lemonde.fr / societe/article/2015/04/29/la-france-veutencadrer-les-jeunes-de-retour-du-djihad_4625129_3224. html

Le Monde (15 de junio de 2015). Manuel Valls: «L'islam est en France pour y rester». Le Monde. Recuperado de http://www. lemonde.fr/religions / article/2015/06/15/manuel-valls1-islam-est-en-france-pour-y-rester_4654354_1653130.html

Le Monde (7 de julio 2015). Islam, voile, immigration... Les désaccords de Rachida Dati avec son camp. Le Monde. Recuperado de http:/ / www.lemonde.fr/politique/article/2015/07/07/ islam-voile-immigration-les-desaccords-de-rachida-datiavec-son-camp_4674054_823448.html

Le Point (13 de enero de 2015). Après les attentats: à Dresde, le mouvement anti-islam exploite la peur. Le point. Recuperado de http://www.lepoint.fr/monde/apres-lesattentats-a-dresde-le-mouvement-anti-islam-exploite-lapeur-13-01-2015-1896049_24.php

Malek, B. (3 de julio de 2015). Génération radicale. Huffington Post $\mathcal{E}$ Le Monde. Recuperado de http://www.huffingtonpost. $\mathrm{fr} / 2015 / 07 / 03 /$ radicalisation-rapport-malek-boutih-felicite-droite-jihadisme_n_7720248.html 
Martinez, J. (19 de octubre de 2015). Le triomphe de la droite ultraconservatrice en Pologne: nationalisme, catholicisme et xénophobie. Révolution Permanente. Recuperado de http:/ / www.revolutionpermanente.fr/Le-triomphe-de-la-droiteultraconservatrice-en-Pologne-nationalisme-catholicismeet-xenophobie

Ministère de l'intérieur (2015). Coup de feu tiré contre une mosquée en construction à Carpentras. Recuperado de http:/ / www.interieur.gouv.fr/Presse/Les-communiques.

Nunès, E. (14 de enero de 2015). Dans les collèges et lycées, le soutien à Charlie Hebdo loin de faire l'unanimité. Le Monde. Recuperado de http://www.lemonde.fr/societe/ article/2015/01/14/a-1-ecole-il-faut-faire-comprendreque-la-laicite-est-notre-fondement_4555679_3224.html

Quillet L. (4 de agosto de 2015). Mais qui était vraiment OrianaFallaci? Le Figaro. Recuperado de http://madame.lefigaro.fr/societe/mais-qui-etait-vraiment-oriana-fallaci-040815-97666

Revue de deux mondes (julio de 2015). Dieu ne vaut pas de moi. Interview confession Michel Houllebecq. Recuperado de http://rddm.revuedesdeuxmondes.fr/archive/article. php? code $=73207 \&$ show $=$ picture .

Rotman, C. (10 de octubre de 2013). On n'est plus chez nous, on est chez eux. Liberation. Recuperado de http:/ / www.liberation.fr/ politiques /2013/10/10/on-n-est-plus-chez-nous-on-estchez-eux_938647

RT (18 de agosto de 2014).15\% of French people back ISIS militants, poll finds. RT. Recuperado de https://www.rt.com/ news/181076-isis-islam-militans-france /

RTL (21 de diciembre de 2014). Le livre de Zemmour, toujours en tête des ventes avec celui de Trierweiler. RTL. Recuperado de http:// www.bfmtv.com/culture/zemmour-en-tete-des-ventesde-livres-mais-peu-lu-853795.html

RTL (16 de noviembre de 2015). Attentats à Paris: "faut fermer les mosquées et les associations qui s'en prennent aux valeurs de la République', dit Manuel Valls". RTL. Recuperado de http:/ /www.rtl.fr/ actu/societe-faits-divers / attentats-a-paris-il-faut-fermerles-mosquees-et-les-associations-qui-s-en-prennent-auxvaleurs-de-la-republique-dit-manuel-valls-7780526167

Simon P. y Tiberj V. (2010). Religions, en Beauchemin, Cris, Christelle 
Hamel et Patrick Simon (coords). Trajectoires et Origines. Enquête sur la diversité des populations en France. Paris: Institut National d'Etudes Démographiques, INSEE.

Sputnik (16 de junio de 2015). Les européens et la montée en puissance des partis d'opposition. Sputnik. Recuperado de http:// fr.sputniknews.com/infographies/20150616/1016561051. html

Todd, E. (2015).Qui est Charlie?: Sociologie d'une crise religieuse. Paris: Seuil.

Walzer, M. (2 de mayo de 2015). Islamism and the Left. Dissident. Recuperado de http://www.dissentmagazine.org/article/islamism-and-the-left

Weber, M. (1959). El político y el científico. México: La Red de Jonas.

Zemmour, E. (2014). Le suicide Français. Paris: Editions Albin Michel. 